\title{
Sleep, neuroengineering and dynamics
}

\author{
Jens Christian Claussen • Ulrich G. Hofmann
}

Received: 23 April 2012/Revised: 28 April 2012/Accepted: 30 April 2012/Published online: 27 May 2012

(C) Springer Science+Business Media B.V. 2012

\begin{abstract}
Modeling of consciousness-related phenomena and neuroengineering are fields that are rapidly growing together. We review recent approaches and developments and point out some promising directions of future research: Understanding the dynamics of consciousness states and associated oscillations, pathological oscillations as well as their treatment by stimulation, neuroprosthetics and braincomputer-interface approaches, and stimulation approaches that probe, influence and strengthen memory consolidation. In all these fields, computational models connect theory, neurophysiology and neuroengineering research and pave a way towards medical applications.
\end{abstract}

Keywords Sleep · Neuroengineering - Dynamics · Computational neuroscience - Brain-computer interface · Neuroprosthetics · Pathological oscillations · Plasticity · Consciousness

Retweeting John von Neumann's words from the 1950s, "All stable processes we shall predict. All unstable processes we shall control.", it becomes immediate how computational neuroscience might form a basis for novel engineering approaches in neural medicine. Brain modeling and neural engineering are, in footsteps of these words,

J. C. Claussen $(\square)$

Institute for Neuro- and Bioinformatics, University of Luebeck, 23538 Lübeck, Germany

e-mail: claussen@inb.uni-luebeck.de

\section{U. G. Hofmann}

Peter-Osypka-Professor for Neuroelectronic Systems, Department for Neurosurgery, Albert-Ludwigs-University Freiburg, Engesserstr. 4, 79108 Freiburg, Germany

e-mail: ulrich.hofmann@uniklinik-freiburg.de aiming at understanding the brain and its emerging phenomena on the scientific side, and interacting with the brain -also with the perspective of medical treatmentfrom the engineering science side. Both sides of the theoryexperiment coin have always been connected as, e.g., electrodes are used for data acquisition as well as to influence neural systems, be it only by well-defined input pulses of a measurement protocol (Siegelbaum and Kandel 1991; Bi and Poo 2001).

This roadmap, however, persistently is a difficult enterprise for various reasons. The brain is a highly nonlinear dynamical system and remains a challenge for data analysis, theoretical modeling, and large-scale computation (Singer 1999; Kantz and Schreiber 1997; Pikovsky et al. 2001; Lehnertz et al. 2000; Olbrich et al. 2011).

In review of these challenges, we would like to emphasize on four areas of research that mark out outstanding future potential, and are related to central keywords: Consciousness, Pathological Oscillations, Neuroprosthetics, and Neuroenhancement. In these four areas, computational models in a similar way connect natural sciences and neuroengineering research and pave a way towards medical applications.

Understanding consciousness, anaesthesia and sleep Large-scale synchronized oscillations are easily observed experimentally and can be both of physiological and pathological character. The mammal sleep-wake cycle is a remarkably robust oscillation, in whose regulation again various neural oscillations are involved, including the cortical slow oscillation in the so-called delta band, with frequencies around $1 \mathrm{~Hz}$ (Compte et al. 2003; Ngo et al. 2010; Mattia and Sanchez-Vives 2012). Electrical stimulation of the brain at this slow wave frequency enhanced the slow oscillations themselves as well as increased their memory 
consolidation effect (Marshall et al. 2006). The slow oscillations, comprised by the interplay between bursting activity and an activity-dependent self-inhibition, exhibit a specific anticorrelation in the durations of Up and Down states, as Mattia and Sanchez-Vives (2012) show in comparison of ferret brain slice data, mean-field models, and simulations. Mean-field models describe the gross activity of a neural subpopulation (at column level or below) but keep track of main types of neurons and their simplified connectivity. They are of great advantage in describing the consciousness transitions of sleep and general anaesthesia (Hutt 2012; Steyn-Ross et al. 2012). As modeled by Hutt (2012), GABAergic tonic inhibition influences the brain's arousal system during general anaesthesia inducing a loss of consciousness. Including the effect of gap junctions on the dynamics elucidated the influence on the propensity of generalized seizures (Steyn-Ross et al. 2012).

Medical treatment: pathological oscillations It is only a recent development that medicine has spotted the essential importance of dynamical phenomena for understanding and treatment of certain diseases. These are primarily those where oscillations themselves comprise the disease as in movement disorders, namely essential tremor and Parkinson's disease, for which electrical stimulation methods co-developed with theoretical work and computer simulations have found their way into clinical practice (Tass et al. 2006). But even when there is no observable mechanical or electrical oscillation, as in the cortical spreading depression which is comprised by slow $\left(10^{2}-10^{3}\right.$ s timescale) $\mathrm{Ca}^{2+}$ waves, control methods may become means of treatment (Dahlem et al. 2008). In epilepsy, and in more severe cases of mood disorders, deep brain stimulation is applied (Abelson et al. 2005) and sophisticated technical implementations as radio stimulation are developed (Delgado et al. 1968). Also, mechanical damages to neural pathways can result in pathological dynamical phenomena, as in the case of spinal cord injury which leads to a hyperexcitability of motorneurons. The modeling approach by Venugopal et al. (2012) goes down to the role of each ion channels and aims at a reduction of spasticity. Finding suitable parameter ranges for electrical stimulation is annother important issue. Krishnamurthi et al. (2012) investigate by measuring velocity reduction how an optimal amplitude of DBS can be found for Parkinson's disease. Considering a Rempe-Terman based computational model of basal ganglia, in Njap et al. (2012) it is demonstrated that a high concentration of the inhibiting neurotransmitter GABA together with electrical stimulation reestablishes faithful thalamocortical relaying. A more general question is addressed in Schütt et al. (2012) by investigating low- to high-frequency stimulation in an Izhikevich-type cortical network model, with the observation that in a frequency range around $100 \mathrm{~Hz}$, a dynamical desynchronization is observed.
Neuroprosthetics and brain-computer interfaces The consequent continuation of few-electrode stimulations and recordings are Human-Machine interfaces (HMIs) (Birbaumer 2006), the most apparent applications where theoretical brain science and engineering meet. In the loss of direct control through the natural pathways, prosthetic devices have to be controlled through an HMI which comprises a "thought-control" (Hochberg et al. 2006; Pfurtscheller et al. 2003). The consequent continuation of that idea-and most immediate demonstration of an HMI at work is that a blind patient can use the HMI for reading (Zrenner et al. 2011), cochlea implants improve hearing (Edgerton et al. 1982), and patients with locked-in syndrome can use an EEG-based BCI for expressing words (Wolpaw et al. 2002). Overall, prosthetic applications require a sound modeling approach and understanding of the neural processing, and, if possible, also coding, to design an effective information interfacing with the brain. The visual system is a part of the brain where experimental research and detailed modeling have made large progress. Here, Norheim et al. (2012) and Einevoll et al. (2012) investigate both a minimal and a feedback-extended model for temporal processing in the lateral geniculate nucleus (LGN). The final step of brain-machine interfacing goes towards dissolution of the border between computer and brain itself: In their beautiful experimental setup, Perez-Marcos et al. (2012) demonstrate virtual hand illusions and-at least for a part of the body-question the conscious awareness of our Self, and thereby connect two quite different fields.

Understanding and influencing neural plasticity Neural plasticity, the basis of all learning, can not only be influenced pharmacologically, but also by various means of electrical stimulation with remarkable effects on cognitive learning and consolidation (Marshall et al. 2006). Stimulation, as well as learning, can effect on both the dynamics (Mattia and Sanchez-Vives 2012; Schütt and Claussen 2012) and on the plasticity (Clopath 2012; Vogt and Hofmann 2012). Vogt and Hofmann (2012) demonstrate modulatory effects of dopamine based on an underlying STDP learning mechanism. Modulatory or multi-input based learning mechanisms are good candidates to explain memory consolidation. In this direction, Clopath (2012) compares two recently proposed mechanisms, namely tagtrigger consolidation, and a metastate tagging model, and provides a comprehensive comparison of both concepts. Finally, Weigenand et al. (2011, 2012) investigate the phase-dependence of stimulation of cortical slow waves. It would be highly interesting, based on improved understanding of neural coding and plasticity mechanisms, to design specific stimulation protocols that selectively strengthen desired acquired memories. 
Outlook While it is tempting to go beyond the purpose of medical treatment by "enhancing the brain" (Farah et al. 2004), a deeper understanding of neural plasticity mechanisms by theoretical and computational models also is expected to offer pathways to the treatment of various neural disabilities and disorders, be them memory-related like Alzheimer, or mood-related disorders as depressive disorders. How emotion modulates learning, and how emotional processes dynamically regulate psychological states is an emerging field (Huber et al. 1998, Figueroa Helland et al. 2008) and can be expected to become the 'neuroengineering' extension of computational modeling for the treatment of brain disorders.

Acknowledgments The collection of articles on these topics in the current and the subsequent issue of Cognitive Neurodynamics emerged from the 1st Baltic Autumn School "Applied Computational Neuroscience: Sleep, Neuroengineering and Dynamics", hosted by the University of Lübeck with generous support from the Deutsche Forschungsgemeinschaft (DFG). We thank all invitees and participants for their contributions for this Special Issue as well as for their enthusiastic and stimulating discussions during the workshop.

\section{References}

Abelson JL, Curtis GC, Sagher O, Albucher RC, Harrigan M, Taylor SF, Martis B, Giordani B (2005) Deep brain stimulation for refractory obsessive-compulsive disorder. Biol Psychiatry 57: $6-510$

Bi G, Poo M (2001) Synaptic modification by correlated activity: Hebb's postulate revisited. Ann Rev Neurosci 24:139-166

Birbaumer N (2006) Brain-computer-interface research: coming of age. Clin Neurophysiol 117:479-483

Clopath C (2012) Synaptic consolidation: an approach to long-term learning. Cogn Neurodyn 6(3):251-257. doi:10.1007/s11571011-9177-6

Compte A, Sanchez-Vives MV, McCormick DA, Wang X (2003) Cellular and network mechanisms of slow oscillatory activity $(<1 \mathrm{hz})$ and wave propagations in a cortical network model. J Neurophysiol 89:2707-2725

Dahlem MA, Schneider FM, Schöll E(2008) Failure of feedback as a putative common mechanism of spreading depolarizations in migraine and stroke. Chaos 18:026110. doi:10.1063/1.2937120

Delgado JM, Mark V, Sweet W, Ervin F, Weiss G, Bach YR, Hagiwara R (1968) Intracerebral radio stimulation and recording in completely free patients. J Nerv Ment Dis 147:329-340

Edgerton BJ, House WF, Hitselberger W (1982) Hearing by cochlear nucleus stimulation in humans. Ann Otol Rhinol Laryngol Suppl 91(2 Pt3):117-124

Einevoll GT, Plesser HE (2012) Extended difference-of-Gaussians model incorporating cortical feedback for relay cells in the lateral geniculate nucleus of cat. Cogn Neurodyn 6(4). doi: 10.1007/s11571-011-9183-8

Farah MJ, Illes J, Cook-Deegan R, Gardner H, Kandel E, King P, Parens E, Sahakian B, Wolpe PR (2004) Neurocognitive enhancement: what can we do and what should we do? Nat Rev Neurosci. 5:421-425

Figueroa Helland VC, Postnova S, Schwarz U, Kurths J, Kundermann B, Hemmeter U, Braun HA (2008) Comparison of different methods for the evaluation of treatment effects from the sleep
EEG of patients with major depression. J Biol Phys 34:393-404. doi:10.1007/s10867-008-9096-x

Hochberg LR et al (2006) Neuronal ensemble control of prosthetic devices by a human with tetraplegia. Nature 442:164-171

Huber MT, Braun HA, Krieg JC (1998) Consequences of deterministic and random dynamics for the course of affective disorders. Biol Psychiatry 46. doi:10.1016/S0006-3223(98)00311-4

Hutt A (2012) The population firing rate in the presence of GABAergic tonic inhibition in single neurons and application to general anaesthesia. Cogn Neurodyn 6(3):227-237. doi: 10.1007/s11571-011-9182-9

Kantz H, Schreiber T (1997) Nonlinear time series analysis. Cambridge University Press, Cambridge

Krishnamurthi N, Mulligan S, Mahant P, Samanta J, Abbas JJ (2012) Deep brain stimulation amplitude alters posture shift velocity in Parkinson's disease. Cogn Neurodyn 6(4). doi:10.1007/s11571012-9201-5

Lehnertz K, Elger CE, Arnhold J, Grassberger P (eds) (2000) Chaos in brain?. World Scientific, Singapore

Marshall L, Helgadottir H, Mölle M, Born J (2006) Boosting oscillations during sleep potentiates memory. Nature 444:610 613

Mattia M, Sanchez-Vives MV (2012) Exploring the spectrum of dynamical regimes and timescales in spontaneous cortical activity. Cogn Neurodyn 6(3):239-250. doi:10.1007/s11571011-9179-4

Ngo HV, Köhler J, Mayer J, Claussen JC, Schuster HG (2010) Triggering up states in all-to-all coupled neurons. EPL Europhys Lett 89:68002. doi:10.1209/0295-5075/89/68002

Njap F, Claussen JC, Moser A, Hofmann UG (2012) Modeling effect of GABAergic current in basal ganglia computational model. Cogn Neurodyn 6(4). doi:10.1007/s11571-012-9203-3

Norheim ES, Wyller J, Nordlie E, Einevoll GT (2012) A minimal mechanistic model for temporal signal processing in the lateral geniculate nucleus. Cogn Neurodyn 6(3):259-281. doi: 10.1007/s11571-012-9198-9

Olbrich E, Claussen JC, Achermann P (2011) The multiple time scales of sleep dynamics as a challenge for modeling the sleeping brain. Phil Trans R Soc A 369:3884-3901. doi:10.1098/ rsta.2011.0082

Perez-Marcos D, Sanchez-Vives MV, Slater M (2012) Is my hand connected to my body? The impact of body continuity and arm alignment on the virtual hand illusion. Cogn Neurodyn 6(4). doi: 10.1007/s11571-011-9178-5

Pfurtscheller G, Mueller GR, Pfurtscheller J, Gerner HJ, Rupp R (2003) 'Thought'-control of functional electrical stimulation to restore hand grasp in a patient with Tetraplegia. Neurosci Lett 351:33-36

Pikovsky A, Rosenblum M, Kurths J (eds) (2001) Synchronization: a universal concept in nonlinear sciences. Cambridge University Press, Cambridge

Schütt M, Claussen JC (2012) Desynchronizing effect of highfrequency stimulation in a generic cortical network model. Cogn Neurodyn 6(4). doi:10.1007/s11571-012-9199-8

Siegelbaum SA, Kandel ER (1991) Learning-related synaptic plasticity: LTP and LTD. Curr Opin Neurobiol 1:113-120

Singer W (1999) Neuronal synchrony: a versatile code for the definition of relations?. Neuron 24:49-65

Steyn-Ross ML, Steyn-Ross DA, Sleigh JW (2012) Gap junctions modulate seizures in a mean-field model of general anesthesia for the cortex. Cogn Neurodyn 6(3):215-225. doi:10.1007/ s11571-012-9194-0

Tass PA, Hauptmann C, Popovych OV (2006) Development of therapeutic brain stimulation techniques with methods from nonlinear dynamics and statistical physics. Int J Bifurcat Chaos 16:1889-1911 
Venugopal RS, Hamm TM, Jung R (2012) Differential contributions of somatic and dendritic calcium-dependent potassium currents to the control of motoneuron excitability following spinal cord injury. Cogn Neurodyn 6(3):283-293. doi:10.1007/s11571-0129191-3

Vogt SM, Hofmann UG (2012) Neuromodulation of STDP through short-term changes in firing causality. Cogn Neurodyn 6(4). doi: 10.1007/s11571-012-9202-4

Weigenand A, Martinetz T, Claussen JC (2011) Switching of cortical up and down states: reproduction of the Shu-HasenstaubMcCormick experiment from a conductance-based model BMC Neuroscience 12, S1, P39. doi:10.1186/1471-2202-12S1-P39
Weigenand A, Martinetz T, Claussen Jens Christian (2012) Phase response of the cortical slow oscillation, submitted

Wolpaw JR, Birbaumer N, McFarland DJ, Pfurtscheller G, Vaughan TM (2002) Brain-computer interfaces for communication and control. Clin Neurophysiol 113:767-791

Zrenner E, Bartz-Schmidt KU, Benav H, Besch D, Bruckmann A, Gabel VP, Gekeler F, Greppmaier U, Harscher A, Kibbel S, Koch J, Kusnyerik A, Peters T, Stingl K, Sachs H, Stett A, Szurman P, Wilhelm B, Wilke R (2011) Subretinal electronic chips allow blind patients to read letters and combine them to words. Proc Biol Sci 278:97-1489 